\title{
Statistics of interacting optical solitons
}

\author{
G. E. Falkovich, ${ }^{1}$ M. G. Stepanov, ${ }^{1,2}$ and S. K. Turitsyn ${ }^{3}$ \\ ${ }^{1}$ Physics of Complex Systems, Weizmann Institute of Science, Rehovot 76100, Israel \\ ${ }^{2}$ Institute of Automation and Electrometry, Novosibirsk 630090, Russia \\ ${ }^{3}$ Photonics Research Group, Aston University, Birmingham B4 7ET, United Kingdom
}

(Received 3 May 2001; published 26 November 2001)

\begin{abstract}
We examine statistics of two interacting optical solitons and describe timing jitter caused by spontaneous emission noise and enhanced by pulse interaction. Dynamics of phase difference is shown to be of crucial importance in determining the probability distribution function (PDF) of the distance between solitons. We find analytically the non-Gaussian tail of the PDF to be exponential. The propagation distance that corresponds to a given bit-error rate is described as a function of system parameters (filtering and noise level), initial distance, and initial phase difference between solitons. We find the interval of parameters where a larger propagation distance can be achieved for higher density of information.
\end{abstract}

DOI: 10.1103/PhysRevE.64.067602

PACS number(s): 42.81.-i, 05.10.Gg, 42.65.Tg, 52.35.Sb

The nonlinear Schrödinger equation (NLSE) is a canonical theoretical model governing propagation of the envelope of a quasimonochromatic wave in a weakly nonlinear dispersive media. Dynamical properties of this generic nonlinear system describing a range of applications from Bose condensate to telecommunications has been thoroughly studied during past few decades. However, statistical problems associated with NLSE are relatively less highlighted in the literature. In this Brief Report we examine a classical problem of soliton interaction in the presence of noise. Though results obtained in this paper can be applied in a variety of physical problems we focus our attention here on optical soliton transmission as an important specific application of the general theory.

In fiber-optic communications, there are two main sources of randomness: variations of system parameters (dispersive, nonlinear and polarization fiber characteristics, amplifier gains, fiber span lengths, and so on) and noise introduced by system devices, the most important is amplified spontaneous emission (ASE) introduced by in-line optical amplifiers. Mathematically, random perturbations of a signal caused by a variety of physical phenomena can be splitted in two main classes, additive and/or multiplicative noise. In traditional optical soliton systems limitations on the error-free transmission are set mainly by stochasticity (and the corresponding arrival jitter) due to the additive ASE noise. Since the noise is usually very weak compared to a signal, one can readily find the (small) deviations of soliton parameters using perturbation theory that is assuming the pulse to be weakly disturbed [1]. However, in general one cannot use perturbation approach to describe the error probability because errors occur when signal changes substantially [2,3]. A priori it is not even clear whether one may still consider signal as solitonlike or fluctuations with a substantial change of the wave form determine the error probability. Large rare fluctuations in a nonlinear system are typically beyond the area of applicability of usual Gaussian statistics [2-4]. On the other hand, neither experiment nor direct numerical simulations are presently able to provide an adequate statistics of such rare errors so that theoretical methods are of utmost importance here. The maximum likelihood approach was suggested in [2] for finding an optimal fluctuation that provides a given large deviation of soliton parameters. The method is technically a saddle-point approximation in the path integral for probabilities and is indeed known to describe the tails of the probability density function [5]. A consistent development of the optimal fluctuation method for soliton-bearing systems has been done in [6] where the conditions on the noise level and propagation distance have been formulated for an optimal fluctuation to be close to a soliton with slowly varying parameters. That made possible to reduce the formally infinitedimensional problem to the analysis of the finite set of soliton parameters and effectively find the error probability for a single soliton transmission under different control schemes [6]. The probability density function (PDF) is essentially Gaussian for timing jitter $[7,8]$ in systems without control and may have substantially non-Gaussian tails in systems with in-line filtering and amplitude modulation [6].

In this Brief Report, we consider interaction of two solitons in the presence of additive noise (action of multiplicative noise has been investigated in [9]). This problem (for lines with filtering) has been first examined in the pioneering paper [2] where phase fluctuations were neglected and some approximation of the optimal path was employed. Here we present a quantitatively accurate description based on the Fokker-Planck equation for the PDF. Such approach was first developed in [10] for systems with in-line filtering using the simple model introduced in [11]. In this Brief Report, we further develop and generalize results of [10] without restricting analysis by lines with filtering. We examine a general problem of transmission both with and without filtering and account for phase dynamics, which is shown below to have serious impact on the error probability. The quantitative results obtained here have also rather transparent physical interpretation. The PDF of the distance between solitons $\mathcal{P}(q)$ has a Gaussian form except for a non-Gaussian (exponential) tail at small distances. Crossover in PDF corresponds to the distance $\bar{q}$ where interaction between solitons is comparable to noise. In other words, since the initial distance is usually larger than $\bar{q}$ then at first stage fluctuations caused by the noise bring solitons to the distance $\bar{q}$. Only 
those solitons will continue to approach each other that have then their phase difference close to zero so that interaction is attractive. The probability density up to an order of unity factor is Gaussian value taken at $\bar{q}$ multiplied by two factors. The first one is the inverse relative speed of solitons (the faster the soliton the smaller the probability to find it at a given point). The second factor is the fraction of $2 \pi$ occupied by the interval of phase differences around zero that guarantees that attraction is not replaced by repulsion on the way from $\bar{q}$ to the final $q$. Both the speed and the phase interval are determined by interaction that exponentially depends on the distance at $q<\bar{q}$. We consider in this Brief Report both a classical problem of two-soliton interaction of the pure nonlinear Schrödinger equation under the action of an additive noise and statistics of interacting solitons in a specific line with a filtering control scheme. We show that the non-Gaussian tail is $\mathcal{P}(q) \propto e^{q}$ in the first case and $\mathcal{P}(q)$ $\propto e^{2 q}$ in the second one. The difference is due to the fact that the relative speed and phase difference are determined by soliton inertia in the pure case and by drag in the filter case. We numerically solve an exact Fokker-Planck equation for probability distribution and find the maximal transmission distance corresponding to a bit-error rate (BER) less than required $\left(<10^{-9}\right)$ level as a function of different system parameters.

The distance $q$ and phase difference $\phi$ between two solitons satisfy the equations written in soliton units [11]

$$
\begin{aligned}
& \ddot{q}+\gamma \dot{q}=-8 e^{-q} \cos \phi+\xi, \\
& \ddot{\phi}+\gamma \dot{\phi}=8 e^{-q} \sin \phi+\xi_{\phi} .
\end{aligned}
$$

All the derivatives are over $z$ (soliton coordinate along the fiber line), $\gamma$ describes the effect of filtering, the noise is white, $\langle\xi(0) \xi(z)\rangle=2 D \delta(z),\left\langle\xi_{\phi}(0) \xi_{\phi}(z)\right\rangle=2 D_{\phi} \delta(z)$, and $D_{\phi}=D\left[3+\gamma^{2}\left(1+\pi^{2} / 12\right)\right] /\left(1+\pi^{2} \gamma^{2} / 4\right)$ [1]. We apply the path-average description valid when the distances are large compared to the amplifier spans. One can neglect the continuous spectrum of perturbations and consider only variations of soliton parameters if noise is weak, $D \ll \sqrt{\gamma}[6]$. For pure Schrödinger case (with $\gamma=0$ ) the continuous spectrum can be neglected if $z$ is less than $D^{-1 / 2}[6,9]$. Since the noise is white one can derive in a usual way the FokkerPlanck equation for the joint $\operatorname{PDF} \mathcal{P}(q, v, \phi, \omega, z)$ with $v$ $=\dot{q}$ and $\omega=\dot{\phi}$

$$
\begin{aligned}
& {\left[\partial_{z}+v \partial_{q}+\omega \partial_{\phi}-\partial_{v}\left(8 e^{-q} \cos \phi+\gamma v\right)\right.} \\
& \left.\quad+\partial_{\omega}\left(8 e^{-q} \sin \phi-\gamma \omega\right)-D \partial_{v}^{2}-D_{\phi} \partial_{\omega}^{2}\right] \mathcal{P}=0 .
\end{aligned}
$$

The initial condition for Eq. (3) is $\mathcal{P}(q, v, \phi, \omega, 0)=\delta(q$ $\left.-q_{0}\right) \delta\left(\phi-\phi_{0}\right) \delta(v) \delta(\omega)$. While we cannot determine analytically $\mathcal{P}(q, v, \phi, \omega, z)$, the distance $\operatorname{PDF} \mathcal{P}(q, z)$ $=\int \mathcal{P}(q, v, \phi, \omega, z) d v d \phi d \omega$ can be effectively described.

Complexity of phase dynamics makes it tempting to consider, following [2], the case $\phi \equiv 0$ neglecting phase fluctuations. Such model, albeit shown unrealistic below, allows one to understand the basic physics involved, it is described

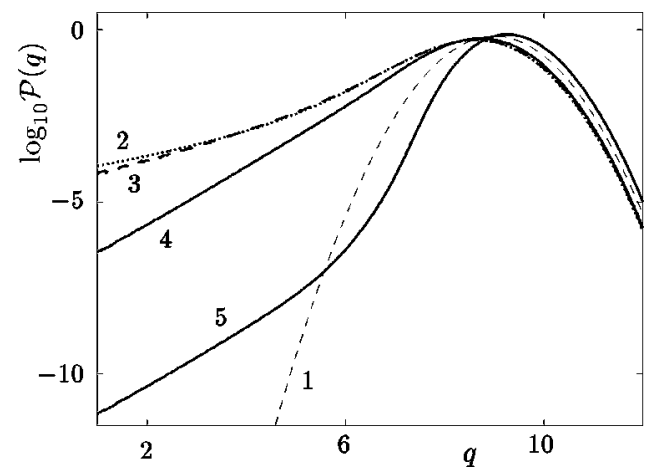

FIG. 1. Distance PDF $\mathcal{P}(q)$. The parameters are as in [2], $q_{0}$ $=9, \gamma=0.4, D=0.0002, Z=150$.

in more details in [10]. At $\phi \equiv 0$, the Eq. (1) gives the following Fokker-Planck equation for the joint $\operatorname{PDF} \mathcal{P}(q, v, z)$,

$$
\left[\partial_{z}+\partial_{q} v-\partial_{v}\left(8 e^{-q}+\gamma v\right)-D \partial_{v}^{2}\right] \mathcal{P}(q, v, z)=0,
$$

with the initial condition $\mathcal{P}(q, v, 0)=\delta\left(q-q_{0}\right) \delta(v)$.

At a sufficiently long time, $\gamma_{z} \gg 1$, the inertia term $\ddot{q}$ can be neglected for all but rare realizations that correspond to the very fast approach of solitons. Neglecting $\ddot{q}$ gives the correct PDF everywhere except a short (order unity) interval of distances where the approximation of exponential interaction breaks anyway. That can be seen in Fig. 1 where curves 2 and 3 presents the solutions of Eqs. (4) and (5) respectively. Without inertia term, Eq. (1) gives the Fokker-Planck equation for $\mathcal{P}(q, z)$

$$
\left[\gamma^{2} \partial_{z}-\gamma \partial_{q} 8 e^{-q}-D \partial_{q}^{2}\right] \mathcal{P}(q, z)=0,
$$

which is exactly solvable in terms of the Whittaker function [10]. For the initial condition $\mathcal{P}(q, 0)=\delta\left(q-q_{0}\right)$, this solution is Gaussian $\mathcal{P}(q) \propto \exp \left[-\gamma^{2}\left(q-q_{0}\right)^{2} / 4 D z\right]$ at $q>\bar{q}(z)$ $\equiv \ln \left(16 z / \gamma q_{0}\right)$ and has an exponential left tail $\mathcal{P}(q) \propto e^{q}$ at $q<\bar{q}(z)$. Effects of noise and interaction are comparable at the distance $\bar{q}(z)$ that can be estimated by substituting the Gaussian distribution into Eq. (5) and equating the second and the third terms. Note that the larger the distance $q$ the weaker the interaction and the closer the PDF to Gaussian. On the contrary, the form of the PDF at $q<\bar{q}$ is indeed completely determined by the balance between filtering and interaction that gives $\dot{q}=-8 e^{-q} / \gamma$. The probability to find a soliton in the interval $(q, q+d q)$ is proportional to time that it spends there, i.e., to $1 / \dot{q} \propto e^{q}$. The shape of the $\operatorname{PDF} \mathcal{P}(q, z)$ at $q<\bar{q}$ does not depend on time, while its amplitude grows [as $\left.\exp \left(-\gamma^{2} q_{0}^{2} / 4 D z\right)\right]$. At $q \ll-2 \ln \gamma$ the velocity is determined by the inertia term, $\dot{q} \propto e^{-q / 2}$, and the tail is $\mathcal{P}(q, z)$ $\propto e^{q / 2}$. The same tail starts from $\bar{q} \approx \ln z^{2} / q_{0}$ at $\gamma z \ll 1$. Indeed, considering Eq. (4) with $\gamma=0$ one sees that the advecting terms (the second and the third) requires $\mathcal{P}(q, v)=\mathcal{P}\left(v^{2}\right.$ $-4 e^{-q}$ ) that after integration over $v$ gives $\mathcal{P}(q, z) \propto e^{q / 2}$.

The first important lesson we thus have learnt from Eq. (4) is that there are two completely different regimes depending on the value of $\gamma z$. At $\gamma z \ll 1$, the effect of filtering can 
be neglected and we have a case of pure nonlinear Schrödinger equation. At $\gamma z \gg 1$, filtering dominates and the inertia terms $\ddot{q}$ and $\ddot{\phi}$ can be neglected in Eqs. (1) and (2) respectively. The second observation is that the form of the left tail of $\mathcal{P}(q, z)$ can be found without the detailed knowledge of the whole PDF. We now apply both these ideas to the complete problem (3). First of all note that $\phi=0$ is an unstable fix point. Even if in-phase solitons are launched $\left(\phi_{0}=0\right)$, the noise necessarily creates a phase difference during propagation, then interaction drives $\phi$ towards the stable point $\phi$ $=\pi$ that corresponds to soliton repulsion. This in particular means that the phase dynamics plays the key role in the statistical analysis and cannot be neglected even in a simplified model. It is also clear that the effect of the phase dynamics must decrease the probability of solitons approaching each other.

We start from the pure case, setting $\gamma=0$. Without interaction, the PDF is Gaussian $\mathcal{P}(q, v, \phi, \omega, z) \propto z^{2} \exp \{-[3(q$ $\left.\left.-q_{q}\right)^{2}-3\left(q-q_{0}\right) v z+v^{2} z^{2}\right] / D t^{3}-\left[3\left(\phi-\phi_{0}\right)^{2}-3\left(\phi-\phi_{0}\right) \omega z\right.$ $\left.+\omega^{2} z^{2}\right] / D_{\phi} t^{3}$ \}. Substituting it into the interaction terms in Eq. (3) one finds that the Gaussian approximations for $P(q, z)$ is valid for $q>\bar{q}=\ln \left(z^{2} / q_{0}\right)$. We assume that, as in the previous cases, noise is unimportant in determining the form of the left tail at $q<\bar{q}$ (it determines only the amplitude). The form of the left tail is determined by interaction. Neglecting the (noise) terms with the second derivatives in Eq. (3) we get the advection-type first-order equation. The characteristics of this equation require the left tail of the $\operatorname{PDF} \mathcal{P}(q, v, \phi, \omega)$ to depend on three (rather than four) variables, $A=4 e^{-q} \cos \phi$ $-v^{2}+\omega^{2}, B=4 e^{-q} \sin \phi+v \omega, \quad$ and $\quad C=\operatorname{Im}(A$ $+i B)^{-1 / 2} \operatorname{arcsinh}(A+i B)^{1 / 2} e^{Q / 2}$, where $Q=q+i \phi$. Note that we exploit here the integrability of nonlinear Schrödinger equation without noise and filtering, in particular, the two-soliton solution (similar to [3]). Indeed, the Eqs. (1) and (2) may be written in a complex form $Q_{z z}=-e^{-Q}$, the above $A, B, C$ are integrals of motion of this equation. Integrating, one gets

$$
\mathcal{P}(q, z)=\int \mathcal{P}(A, B, C, z) d v d \phi d \omega \propto e^{-q} .
$$

Note that even though the velocity of the approaching solitons behaves as $v \propto e^{-q / 2}$ (as it did at $\phi \equiv 0$ ) the PDF is not proportional to $v^{-1}$. The reason is that phase dynamics makes any nonzero phase difference to go beyond the interval $[-\pi / 2, \pi / 2]$ turning interaction into repulsion. That requires the phase difference to be close to zero when the distance passes through $\bar{q}$ in order to reach some smaller $q$ eventually. This brings extra small factor $\exp [(q-\bar{q}) / 2]$ into the PDF. Formally, it comes because the domain of integration over $C$ in Eq. (6) is exponentially restricted. As a result, the left tail $\mathcal{P}(q) \propto e^{q}$ does not correspond to a constant flux $\dot{q} \mathcal{P}$ as it was without accounting for phase dynamics.

Next we analyze systems with filtering in the limit $\gamma z$ $\gg 1$. Neglecting soliton inertia one obtains the following Fokker-Planck equation for the joint $\operatorname{PDF} \mathcal{P}(q, \phi, z)$,

$$
\begin{aligned}
& {\left[\gamma^{2} \partial_{z}+\left(\partial_{\phi} \sin \phi-\partial_{q} \cos \phi\right) 8 \gamma e^{-q}-D \partial_{q}^{2}-D{ }_{\phi} \partial_{\phi}^{2}\right]} \\
& \quad \times \mathcal{P}(q, \phi, z)=0 .
\end{aligned}
$$

As it was discussed above at $q<\bar{q}$ interaction dominates and one may disregard the two last (noise) terms and find out that $\mathcal{P}(q, \phi, z) \propto e^{2 q}$ turns the second (advective) term in Eq. (7) into zero. One factor $e^{q}$ comes from velocity and another from phase. Indeed, to pass from $\bar{q}$ to some smaller $q$, one needs an attraction (in other terms $\phi<\pi / 2$ ). This implies that the phase at $q \simeq \bar{q}$ is exponentially small since the trajectories in $\phi-q$ plane are exponential near zero phase, $\phi$ $\propto e^{-q}$. That contributes the factor $\exp (q-\bar{q})$ to the probability. At $q>\bar{q}$, the PDF fits the Gaussian distribution. To verify these results and to obtain quantitative description of the PDF we numerically solve Eq. (7).

Figure 1 compares distance PDFs obtained by different methods. Curve 1 is Gaussian obtained without interaction. Curve 2 presents an accurate computation of the model from [2], that is the numerical solution of Eq. (4). It is seen that the PDF is qualitatively similar but the error probability has been substantially underestimated in [2]. This happened because of two reasons: first, a true optimal fluctuation is different from suggested in [2] and, second, one needs the account of fluctuations around the optimal one to get a correct form of the non-Gaussian PDF tail (more details can be found in [10]). Curve 3 presents the solution of Eq. (5), one can see that inertia can be indeed neglected for all practical purposes. Account of phase fluctuations, that is solving Eq. (7), gives curves 4 and 5 for the initial phases $\phi_{0}=0$ and $\phi_{0}=\pi$, respectively. They both follow $\mathcal{P}(q) \propto e^{2 q}$ at $q \leqq 6$. $\mathcal{P}(q)$ is equal to $v^{-1} \propto e^{q}$ multiplied by the width of the phase interval starting from which at $q_{0}=\bar{q}$ we reach coordinate $q$. The latter factor is proportional to $e^{q}$ as can be readily derived from the system $\gamma \dot{q}=-8 e^{-q} \cos \phi, \gamma \dot{\phi}$ $=8 e^{-q} \sin \phi$. Note that in this region curve 4 indeed gives $\mathcal{P}$ that is $\exp (q-\bar{q})$ times smaller than that given by curve 3 . We see that phase fluctuations dramatically decrease the probability of solitons approaching each other. Note also that choosing initial phase $\phi_{0}=\pi$ (corresponding to repulsion) allows one to reduce the probability even further. Note that

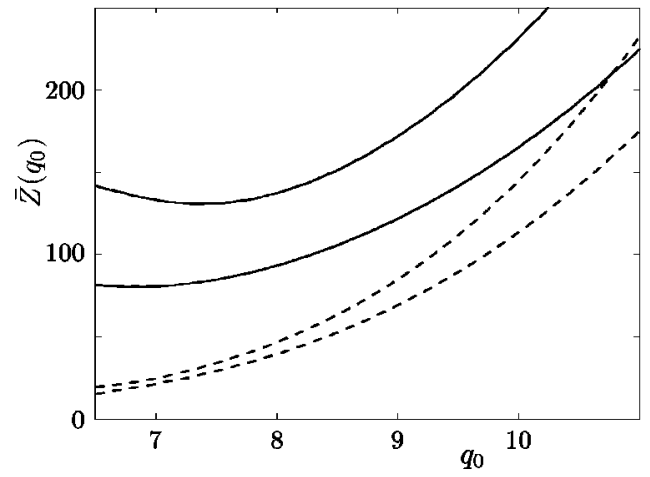

FIG. 2. Error-free distance as function of initial spacing. $D$ $=0.0002$ and $D=0.0003$ (upper and lower curves), $\phi_{0}=\pi$ and $\phi_{0}=0$ (solid and dashed curves), $\gamma=0.4$. 


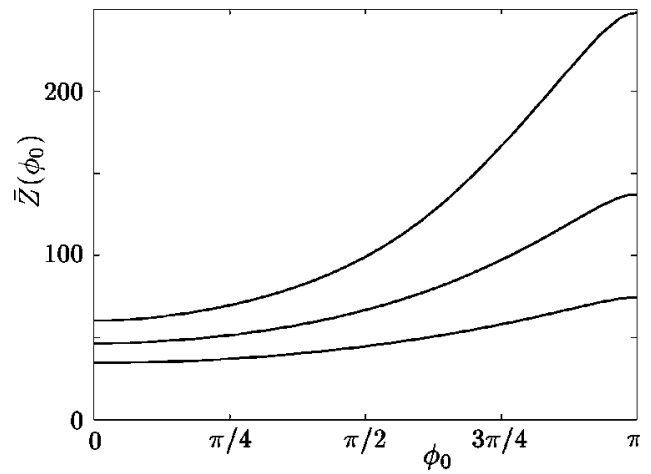

FIG. 3. Error-free distance as function of initial phase difference. $D=0.0004, D=0.0002$, and $D=0.0001$ (from the bottom to the top). $q_{0}=8, \gamma=0.4$.

in the filtering case the phase dynamics makes the left tail fluxless as it does in the pure case. Indeed, the solutions of Eqs. (4) and (5) do not conserve probability since their left tails correspond to a constant flux $\dot{q} \mathcal{P}(q)$ towards small $q$. On the contrary, phase dynamics returns solitons back to large distances and the flux decreases as $q$ decreases on the solution of Eq. (7).

To link our theoretical analysis with the practical issue of evaluation of the transmission system performance we ought to estimate the contribution from the process considered to the so-called BER, defined as the probability of incorrect identification of a bit in the transmitted data stream. To calculate BER one has to specify a receiver. Here we assume that the signal-to-noise ratio is large enough and the main contribution to BER results from soliton interaction. More specifically, we assume that there is an error when the solitons approach each other closer than the threshold $q_{Z}$, the respective contribution to the bit-error rate $E\left(q_{T}, q_{0}, \phi_{0}, Z ; \gamma, D\right)=\int_{0}^{q_{Z}} d q \mathcal{P}(q, Z)$ as a function of all six parameters was studied in [10]. In this Brief Report, we consider without loss of generality the most "dangerous" case when two solitons are surrounded by empty (zero) slots. We assume that the error occurs when the distance between solitons is either smaller than $q_{0} / 4$ or larger than $7 q_{0} / 4$. Such contribution to the BER (we call it here $\mathrm{BER}_{1}$ ) is calculated according to the formula $E\left(q_{0}, \phi_{0}, Z ; \gamma, D\right)=1$ $-\int_{q_{0} / 4}^{7 q_{0} / 4} d q \mathcal{P}(q, Z)$.

For practical purposes, it is BER that imposes the restrictions on the system parameters. We find here the propagation distance $\bar{Z}$ (error-free distance) that corresponds to the standard value $\mathrm{BER}_{1}=10^{-9}$. Apparently, $\bar{Z}\left(q_{0}, \phi_{0} ; \gamma, D\right)$ depends on four parameters. We present the results as the dependencies on the initial parameters $q_{0}, \phi_{0}$ taken at different values of the noise level $D$; see Figs. 2 and 3. The most interesting are two upper curves in Fig. 2 that show the dependence $\bar{Z}\left(q_{0}\right)$ for $\phi_{0}=\pi$. Note that the curves contain a part where $\bar{Z}$ increases when $q_{0}$ decreases. That is because the main contribution to $\mathrm{BER}_{1}$ at those parameters are made by the events that correspond to soliton approach. Since at the beginning $\phi_{0}=\pi$ then it takes more time for noise to overcome interaction and reach $\phi<\pi / 2$ when $q_{0}$ is smaller. Surprisingly, larger propagation distance can be achieved for higher density of information in this interval of parameters.

To conclude, we have developed an original analytical method to describe the non-Gaussian tail of the probability distribution of the distance between interacting solitons and numerically obtained the whole distribution. We have described how soliton interaction enhances the effect of noise and increases the probability of two solitons to approach each other.

We thank V. Lebedev and I. Gabitov for useful discussions. This work was supported by Minerva Foundation.
[1] E. Iannone, F. Matera, A. Mecozzi, and M. Settembre, Nonlinear Optical Communication Networks (Wiley, New York, 1998).

[2] C. R. Menyuk, Opt. Lett. 20, 285 (1995).

[3] T. Georges, Opt. Commun. 123, 617 (1996).

[4] F. Kh. Abdullaev, S. A. Darmanyan, and F. Lederer, Opt. Commun. 126, 89 (1996).

[5] G. Falkovich, I. Kolokolov, V. Lebedev, and A. Migdal, Phys. Rev. E 54, 4896 (1996).
[6] G. Falkovich, I. Kolokolov, V. Lebedev, and S. Turitsyn, Phys. Rev. E 63, 025601(R) (2001).

[7] J. P. Gordon and H. A. Haus, Opt. Lett. 11, 665 (1986).

[8] J. Elgin, Phys. Lett. 110A, 441 (1985).

[9] M. Chertkov, I. Gabitov, I. Kolokolov, and V. Lebedev, Pis'ma Zh. Exp. Teor. Fiz. 74, 391 (2001).

[10] G. Falkovich and M. G. Stepanov, Opt. Lett. (to be published). [11] J. P. Gordon, Opt. Lett. 8, 596 (1983). 